\title{
Peer Perceptions and Social Support for Young Adults on the Cancer Continuum
}

\author{
Emily Lehman \\ Dominican University of California \\ IRB 10830
}

\section{Introduction}

From processing a cancer diagnosis to treatment, social support is essential and beneficial to the wellbeing of young adults with cancer (Breuer et al., 2017).

Young adults on the cancer continuum report a sense of social isolation due to a lack of understanding among peers about their experiences and diagnoses (lannarino et al., 2017).

Social support is given online rather than in person due to the positive language and communication that relies on the written word more than social cues (Warner et al., 2018).

\section{Hypotheses}

- Young adults will show more in person and virtual support to a person who is in remission than a peer who is in curren treatment of cancer

- Young adults will be more socially attracted to a peer who is in remission than a peer who is in current treatment for cancer. - Young adults will show more social support to peers virtually than in person.

\section{Methods}

The study utilized a sample of 152 young adults (18-25 years old) with a mean age of 20.77. 30 participants were male and 151 participants were female, with one participant preferring not to say. $60 \%$ of the sample identified as being White/Caucasian.

Participants were asked to complete an online survey after reading a vignette about a peer who is in treatment or remission of cancer including questions about social attractiveness, types of support and the following two measures:

The Measure of Interpersonal Attraction- Social Attraction Subscale (McCroskey \& McCain, 1974) measuring overall peer social attractiveness on a 5-point Likert Scale.

Virtual and In Person Support Measure measuring type of

support given to a peer on a 5-point Likert Scale.

- I would send a supportive text to Kate

- I would bring flowers to Kate

\section{Results}

In comparing the mean scores between participants who read the vignette about a peer who is in treatment of cancer and the vignette about a peer who is in remission of cancer:

- No significant difference was found in their amount of social support given to a peer who is in remission of cancer and peer who is in treatment of cancer $(t(150)=-1.358, p>0.05) ;(t(150)=-0.488, p>$ 0.05)

- Significant difference was found in their perception of social attractiveness of peer who is in remission of cancer and a peer who is in treatment of cancer $(\mathrm{t}(150)=2.474, \mathrm{p}<0.05$ )

- No significant difference was found in their types of social support, in person and virtual, given to a peer on the cancer continuum $\quad(t(151)=$ $.996, p>0.05$

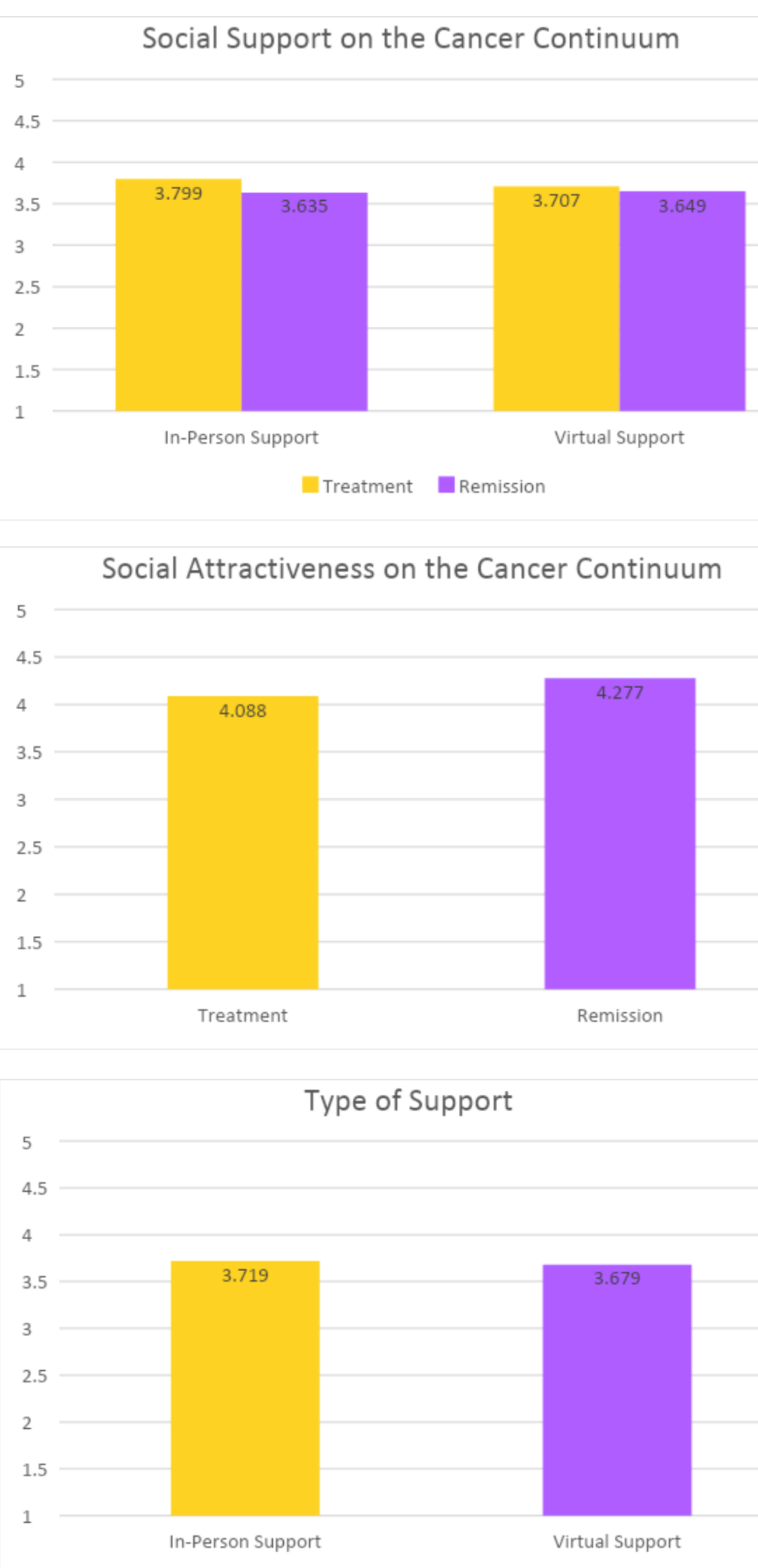

DOMINICAN UNIVERSITY

\section{Discussion}

The purpose of this study was to analyze the different types of support given to a peer on the cancer continuum, and the overall perception of social attractiveness of that peer.

The results showed that young adults do not prefer to give more support to a peer in remission of cancer rather than a peer who is in current treatment of cancer.

The results however did show that young adults perceived a peer in remission of cancer to be more socially attractive than a peer who is in current treatment of cancer. This supports previous research that perceived weakness of a young adult in treatment of cancer makes them less socially attractive than a peer who is in remission (Mosher \& Danoff-Burg, 2009).

The results also did not show that peers would give more virtual support than in-person support to a peer on the cancer continuum.

\section{Limitations}

- Age range of participants was not the traditional young adult age range, 18-35 years old

- The vignette's usage of the word 'peer'

- Gender may play a role in young adults support for a peer on the cancer continuum

\section{Future Directions}

- Future studies should broaden the age range of participants to the traditional young adult age range, 18-35 years old.

- Research peer perceptions of social attractiveness and amounts of social support given to a peer on the cancer continuum among adolescents, $10-19$ years old.

- Analyze the difference between perceptions of social attractiveness of a peer who is healthy and a peer on the cancer continuum. 